\title{
Alterations of White Matter Integrity in Cerebral Small Vessel Disease and Their Correlation with Cognitive Performance: A Trace-Based Spatial Statistics Study
}

\section{Yifan Wang}

E.g. Shanghai Fifth People's Hospital, Fudan University

\section{Tianyao Wang}

E.g. Shanghai Fifth People's Hospital, Fudan University

\section{Zekuan Yu}

E.g. Academy for Engineering and Technology, Fudan University

\section{Bo Huang}

E.g. Guigang City People's Hospital

Biao Liu

E.g. Guigang City People's Hospital

Xianwei Liu

E.g.Tongren Hospital, Shanghai Jiao Tong University School of Medicine

Huabin Yin

E.g. Shanghai Fifth People's Hospital, Fudan University

Jun Liu( $\sim$ lj7275@163.com )

E.g.Tongren Hospital, Shanghai Jiao Tong University School of Medicine

\section{Research Article}

Keywords: cerebral small vessel disease, white matter hyperintensities, white matter microstructure, cognition, trace-based spatial statistics

Posted Date: January 7th, 2021

DOl: https://doi.org/10.21203/rs.3.rs-134624/v1

License: (9) (1) This work is licensed under a Creative Commons Attribution 4.0 International License. Read Full License 


\section{Alterations of White Matter Integrity in Cerebral Small Vessel}

\section{Disease and Their Correlation with Cognitive Performance: A}

\section{Trace-Based Spatial Statistics Study}

Yifan Wang ${ }^{\mathrm{a} 1}$, Tianyao Wang ${ }^{\mathrm{a} 1}$, Zekuan Yu ${ }^{\mathrm{defg} 1 *}$, Bo Huang ${ }^{\mathrm{b}}$, Biao Liu ${ }^{\mathrm{b}}$, Xianwei Liuc ${ }^{\mathrm{c}}$, Huabin Yin a , Jun $\mathrm{Liu}^{\mathrm{c} *}$

${ }^{a}$ Department of Radiology, Shanghai Fifth People's Hospital, Fudan University, Shanghai, China

${ }^{b}$ Department of Radiology, Guigang City People's Hospital, Guigang, China

${ }^{c}$ Department of Radiology, Tongren Hospital, Shanghai Jiao Tong University School of Medicine, Shanghai, China

${ }^{d}$ Academy for Engineering and Technology, Fudan University, Shanghai, 200433, China

${ }^{e}$ Key Laboratory of Industrial Dust Prevention and Control \& Occupational Health and Safety, Ministry of Education

${ }^{f}$ Anhui Province Engineering Laboratory of Occupational Health and Safety

${ }^{g}$ Laboratory of Industrial dust deep reduction and occupational health and safety of Anhui Higher Education Institutes

* Corresponding authors:

Zekuan Yu, assistant professor, Academy for Engineering and Technology, Fudan University, 539 Handan Road, Shanghai, 200336, China; E-mail: yzk@fudan.edu.cn Or

Jun Liu, Prof, Department of Radiology, Tongren Hospital, Shanghai Jiao Tong University School of Medicine, 1111 XianXia Road, Shanghai, 200336, China; E-mail: Ij7275@163.com

\section{Abstract}

Background: This study aimed to understand the injury of white matter (WM) microstructure behind white matter hyperintensities (WMH) and identify the regions where injury was more pronounced with increasing WMH severity. Moreover, we analyzed whether this microstructural injury is related to cognition.

Methods: 110 patients with WMH were recruited in this research. All subjects underwent 3.0T MRI scans and neuropsychological cognitive assessments. Simple mental state examination (MMSE) along with Montreal Cognitive Assessment (MoCA) were applied to assess the patient's overall cognitive ability. WMH of each subject was graded according to Fazekas grade scale and was 
divided into two groups: (A) WMH score of 1-2 points (n=64), (b) WMH score of 3-6 points $(n=46)$. Trace-based spatial statistics (TBSS) was applied for the analysis of diffusion tensor imaging (DTI) data. All statistical analyses were performed in SPSS 26.0 statistical software.

Results: The results indicate that patients with higher WMH scores showed extensively symmetrical areas of increased mean diffusion, axial diffusion and radial diffusion involving bilateral anterior limb, posterior limb and retrolenticular part of internal capsule, posterior corona radiata, external capsule, superior longitudinal fasciculus, and superior fronto-occipital fasciculus $(\mathrm{P}<0.01)$.

Conclusions: Finally, we come to the conclusion that cognition-related WM fiber tracts tend to be more vulnerable to be injured in patients of cerebral small vessel disease (CSVD). Moreover, changes in WM microstructure often predate changes of cognition. Early detection of microstructural changes and timely intervention can delay cognitive decline to some extent.

Keywords: cerebral small vessel disease; white matter hyperintensities; white matter microstructure; cognition; trace-based spatial statistics

\section{Introduction}

Cerebral small vessel disease (CSVD) is a group of clinical syndromes involving cerebral arterioles, microarteries, capillaries and venules, which accounts for approximately $10 \%-30 \%$ of global ischemic strokes[1] and is a major vascular contributor to cognitive deficits and dementia[2].

White matter hyperintensity (WMH), as one the common imaging markers of CSVD has been widely reported to be associated with cognitive decline and progression of cognitive impairment[35]. As a matter of fact, WMHs observed on conventional Magnetic resonance imaging (MRI) are only the tip of the iceberg of CSVD-related injuries. Knowing the changes of the microstructure 
behind WMH is important to exactly understand the characteristic and severity of white matter (WM) injury as well as the mechanism of WMH-related cognitive impairment.

Diffusion tensor imaging (DTI) is an advanced technique for detecting changes in the microstructure of WM[6], which is sensitive to the change of WM microstructure integrity[7, 8]. It can not only reflect the injury of WM in WMH area, but also detect the change of WM fiber tracts which seem normal on traditional MRI[9]. Relevant studies have shown that injury of WM microstructure is related to cognitive impairment $[10,11]$. Therefore, DTI can be used to explore the characteristic of WM injury at the micro level and the neural mechanism of cognitive impairment caused by WMH. Under usual circumstances, four main diffusion indicators including fractional anisotropy (FA), mean diffusion (MD), axial diffusion (AD) and radial diffusion (RD) are applied to provide more information on WM microstructure and its changes in relation to cognitive function [12].

Among different methods used in DTI research, trace-based spatial statistics (TBSS) is a reliable and optimized one that minimizes registration errors and personal evaluation biases, and is considered to improve sensitivity, objectivity, and interpretability when applied to multiple diffused data[13]. To our knowledge, there have been a number research on the change of WM microstructural integrity in WMH patients and its correlation with cognition[14-17]. However, few TBSS studies have directly identified differences in diffusion measurements between patients with varying degrees of WMH. Little is known about how the microstructure of WM changes at the local level as the severity of WMH increases and whether it is related to cognition.

In this article, we aim at understanding the injury of the WM microstructure behind WMH and identifying the regions where injury was more pronounced with increasing WMH severity. 
Moreover, we analyzed whether this microstructural injury is related to cognition.

\section{Materials and methods}

\section{Participants}

110 patients with WMH were recruited from Tongren Hospital, Shanghai Jiao Tong University School of Medicine, China. The diagnosis of WMH was visualized by two radiologists who evaluated the MR fluid attenuation inversion recovery (FLAIR) sequence image without knowing the clinical data of the subject. Each subject received a standard baseline assessment, including complete sociodemographic and clinical data vascular risk factors (VRF), neuropsychological assessment and multimodal MRI. The inclusion criteria were as follows: 1) patients aged older than 55 years, 2) no history of brain trauma or dementia, 3) MRI scan showed WMH imaging manifestations. The exclusion criteria were as follows: 1) non-lacunar infarction in cerebral cortex or cerebellum or brainstem, 2) have a history of hydrocephalus, cerebral tumor or space occupation, 3) unable to cooperate with this study independently or suffering from serious physical and mental diseases, 4) MRI contraindications, 5) leukodystrophy caused by other causes (such as multiple sclerosis, history of brain exposure, etc)

According to Fazekas grade scale[18], WMH in the patient's periventricular and deep white matter were graded separately, and the two grades were added together to record the total score. Finally, WMH patients were divided into two groups:(A) WMH score of 1-2 points, (b) WMH score of 3-6 points.

\section{Neuropsychological assessment}

In this study, all subjects underwent neuropsychological cognitive assessment within one week 
of MRI examination. We performed a simple mental state examination (MMSE) along with Montreal Cognitive Assessment (MOCA) for cognitive assessment and recorded the total score.

\section{MRI acquisition}

All the subjects were scanned by Siemens 3Tesla Skyra scanner (Siemens, Germany). An twentychannel standard head coil with foam pads is used to limit head movement. 3D T1-weighted MPRAGE with TR/TE/TI $=2,400 / 2.13 / 1100 \mathrm{~ms}, \mathrm{FOV}=256 \times 256 \mathrm{~mm}^{2}$, and number of slices $=192.3 \mathrm{D}$ T2W-FLAIR with TR/TE/TI $=5000 / 395 / 1800 \mathrm{~ms}, \mathrm{FOV}=256 * 256 \mathrm{~mm}^{2}$, and number of slices $=192$. DTI with $\mathrm{TR} / \mathrm{TE}=8300 / 74 \mathrm{~ms}, \mathrm{FOV}=256 \times 256 \mathrm{~mm}^{2}$, number of slices $=192$, and 30 diffusion weighted scans with $\mathrm{a} b$ value of $1000 \mathrm{~s} / \mathrm{mm}^{2}$. MRI data was evaluated by two radiologists who had no knowledge of the clinical information.

\section{Image preprocessing}

The steps of the DTI data preprocessing were as follows [19]: (1) Use the nonlinear image registration tool of FMRIB to affine align each diffusion-weighted volume with the corresponding b0 image and to correct possible motion artifacts and eddy current distortion. (2) The fractional threshold of 0.2 was applied to remove brain tissue. (3) The DTIfit within FSL was used to create FA, MD, AD, and RD images at each brain voxel. All the steps were performed on the Functional MRI of the Brain Software Library (FSL) version 5.0.9 (http://fsl.fmrib.ox.ac.uk).

\section{Tract-Based Spatial Statistics (TBSS)}

Firstly, FSL nonlinear image registration algorithm was used to align the FA map of each subject to FMRIB58_FA standard space. Then, the mean FA image is generated. By refining the mean FA image, the mean FA skeleton representing the core structure of WM domain is generated later. Finally, individual subject FA images were projected onto the mean FA skeleton. These skeleton 
121

123

124 data as well as neuropsychological data. All relevant results are depicted in Table 1.

137 Table 1 Demographic, clinical characteristics and neuropsychological data

\begin{tabular}{cccc}
\hline Items & Group A(n=64) & Group B(n=46) & p-value \\
\hline Age & $65(7)$ & $69(13)$ & $0.001^{\text {a }}$ \\
Female, n (\%) & $44(68.7)$ & $33(71.1)$ & 0.736 \\
Hypertension, n (\%) & $24(37.5)$ & $13(31.7)$ & 0.264 \\
Diabetes, n (\%) & $40(76.9)$ & $29(82.9)$ & 0.348 \\
hyperlipemia, n (\%) & $31(56.4)$ & $20(57.1)$ & 0.752 \\
TC & $4.52(1.08)$ & $4.38 \pm 0.16$ & 0.586 \\
TG & $1.21(0.64)$ & $1.50(0.98)$ & 0.199 \\
HDL & $1.37 \pm 0.56$ & $1.27 \pm 0.69$ & 0.140
\end{tabular}

\section{Statistical analysis}

All statistical analyses were performed in SPSS26.0 statistical software [20]. Demographic, clinical characteristics, medical history, and neuropsychological data were compared by $\mathrm{t}$ test, chi square test, and nonparametric test. We used t-test to compare the difference of DTI-derived indexes between the two groups. In order to control class I errors, false discovery rate (FDR) correction is adopted. Then, linear regression analysis was used for age correction. $\mathrm{P}<0.01$ was considered statistically significant [21]. Partial correlation analysis was used to evaluate the relationship between DTI-derived indexes and overall cognitive function. Age, gender, and education level were considered as covariates in partial correlation analysis. $\mathrm{P}<0.05$ was considered statistically significant [22].

\section{Results}

In terms of demographic data, no significant difference exists between two groups except the age $(p<0.05)$. Compared to subjects in Group A, subjects in Group B were characterized by an older mean age significantly. In addition, no statistically difference was observed on the aspect of clinical

(1)

$1.27 \pm 0.69$ 


\begin{tabular}{cccc} 
LDL & $2.85 \pm 0.10$ & $2.61(1.42)$ & 0.276 \\
MMSE & $29.00(1.00)$ & $29.00(2.00)$ & 0.091 \\
MOCA & $24.00(5.00)$ & $24.00(5.00)$ & 0.095 \\
\hline
\end{tabular}

\section{TBSS Analysis}

After age correction, compared to the group of low WMH scores, the patient group with high WMH scores showed extensively symmetrical areas of increased MD and RD involving the bilateral anterior limb of internal capsule, bilateral posterior limb of internal capsule, bilateral retrolenticular part of internal capsule, bilateral superior corona radiata, bilateral posterior corona radiata, bilateral external capsule, bilateral superior longitudinal fasciculus, bilateral superior fronto-occipital fasciculus, and bilateral anterior corona radiata $(\mathrm{P}<0.01, \mathrm{FDR}$ corrected). Increased $\mathrm{AD}$ was present in the above areas except the bilateral superior corona radiata as well as bilateral anterior corona radiata $(\mathrm{P}<0.01$, FDR corrected) and the results are shown in the Figure. 1. We also found decreased FA in the bilateral superior fronto-occipital fasciculus, right posterior limb of internal capsule and left posterior corona radiata, where increased $\mathrm{MD}, \mathrm{RD}$ and $\mathrm{AD}$ were present $(\mathrm{P}<0.01$, FDR corrected), the results are shown in the Figure. 2. Besides, decreased FA were observed in the bilateral tapetum along with left posterior thalamic radiation $(\mathrm{P}<0.01$, FDR corrected $)$, the results are shown in the Figure. 3.

\footnotetext{
1 Values with normal distribution are presented as the mean \pm stand deviation (SD); Values with nonnormal distribution are presented as median (interquartile range).

${ }^{\mathrm{a}}$ : The difference between groups was statistically significant $(\mathrm{p}<0.01)$

TC: total cholesterol; TG: triglyceride; HDL: high density lipoprotein; LDL: low density lipoprotein; MMSE: mini-mental state examination; MoCA: montreal cognitive assessment
} 
153
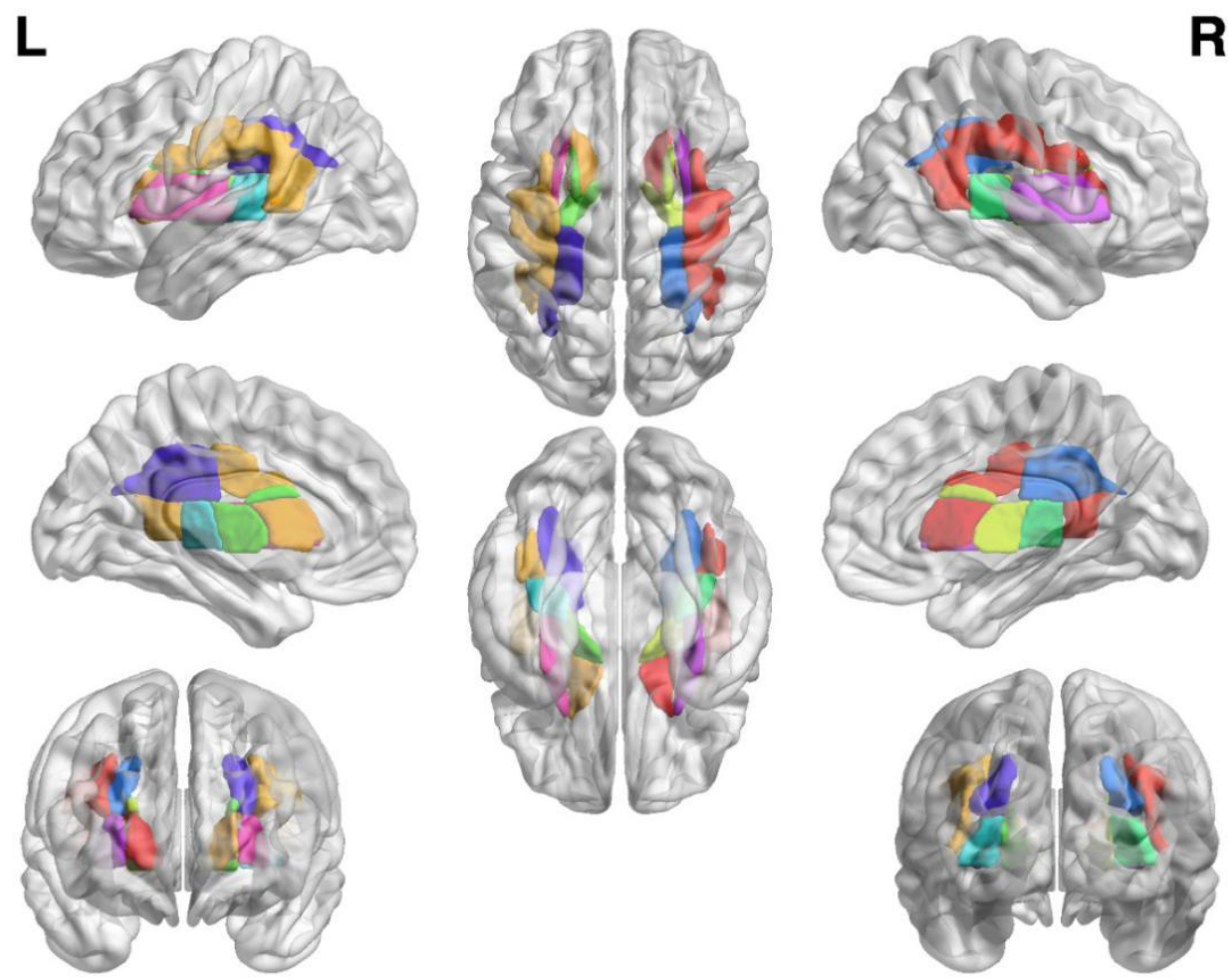

Fig. 1 Different axial diffusion (AD) values of white matter fiber bundles between two groups are marked in colors. Patients in Group B had higher AD values of some white matter fiber bundles than those in group A.
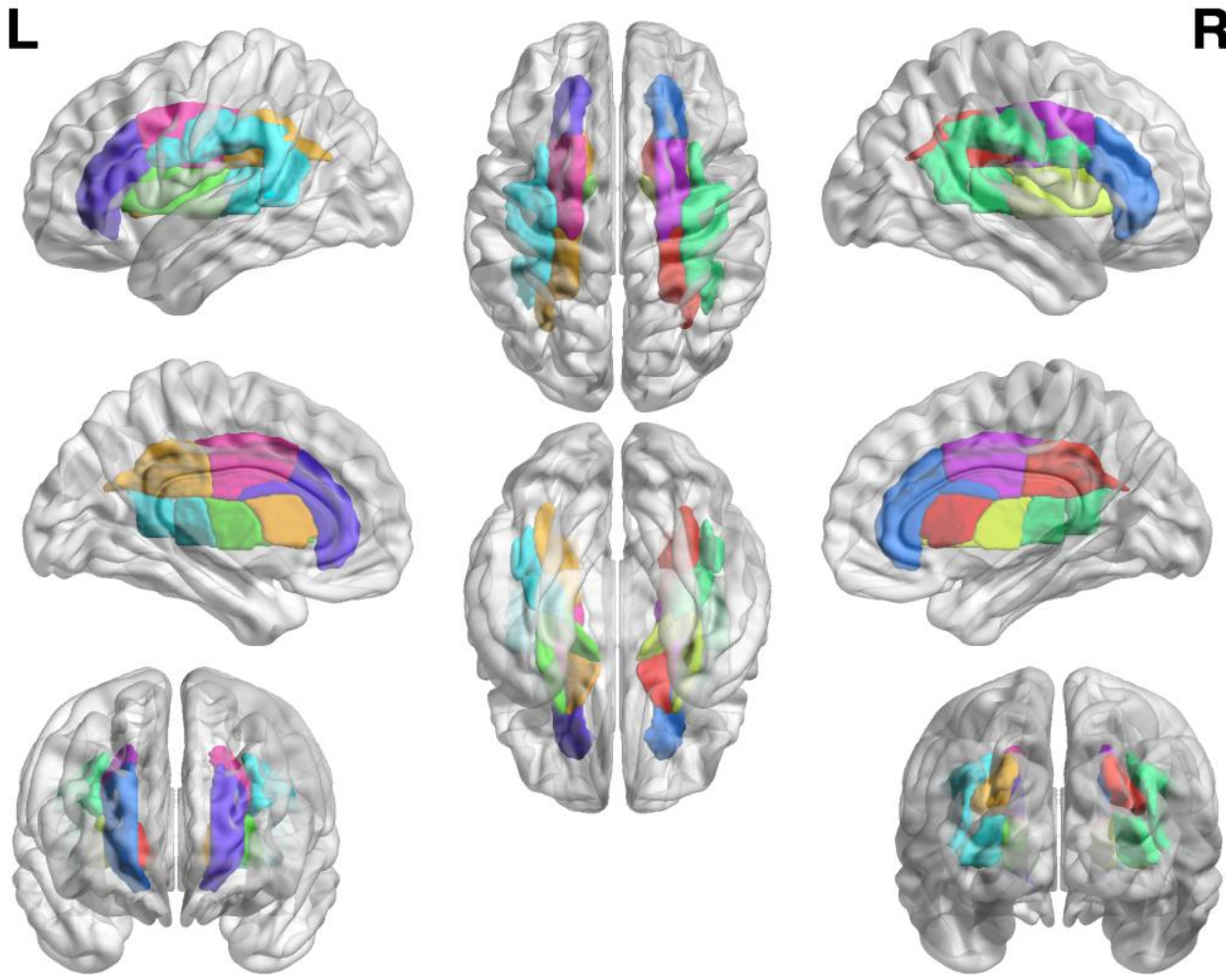

Fig. 2 Different mean diffusion (MD) and radial diffusion (RD) values of white matter fiber bundles between two groups are marked in colors. Patients in Group B had higher MD and RD values of some white matter fiber bundles than those in group $A$. 

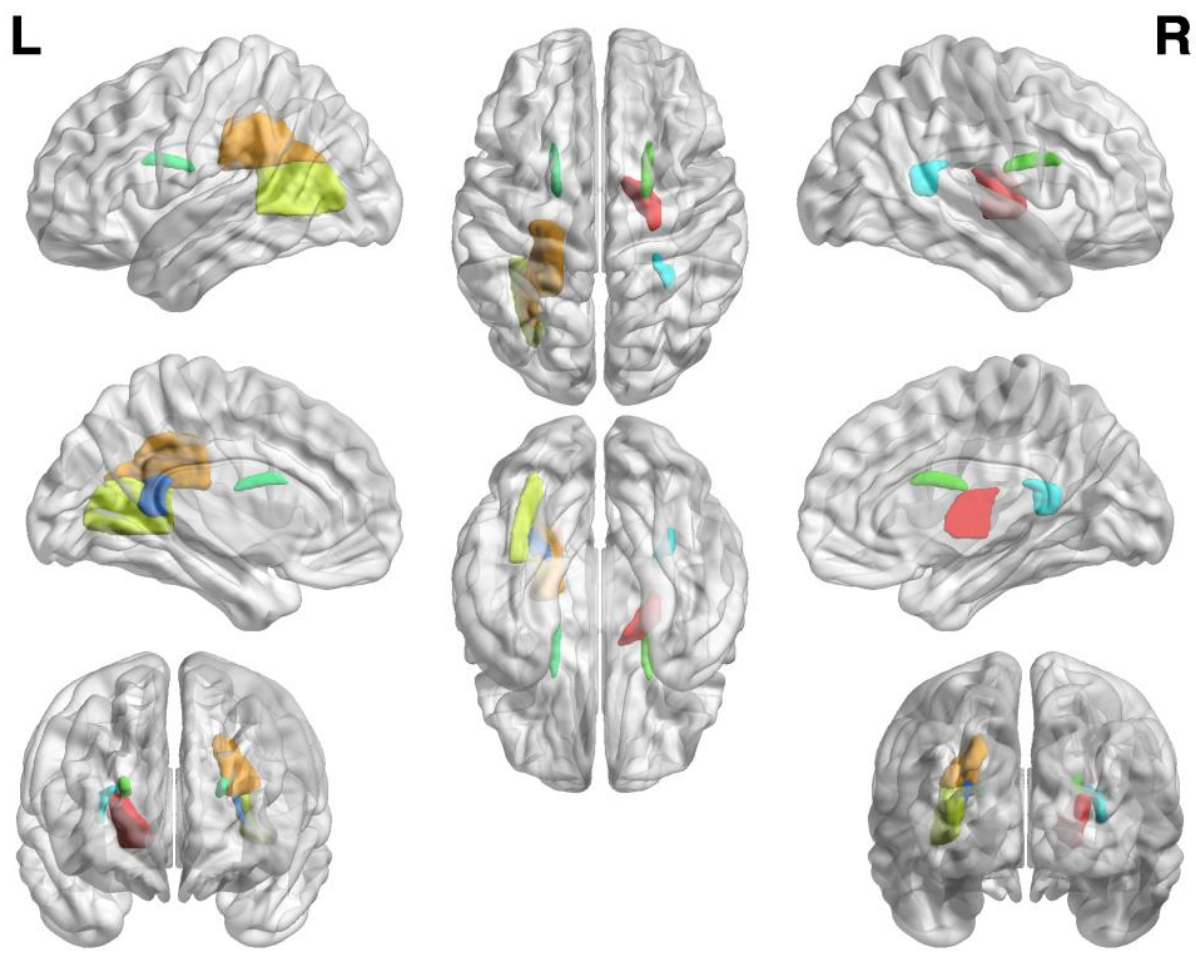

Fig. 3 Different fractional anisotropy (FA) values of white matter fiber bundles between two groups are marked in colors. Patients in Group B had lower FA values of some white matter fiber bundles than those in group A.

\section{Correlation Between DTI Measures and Cognitive Performance}

During our research, no significant relationship was observed between the four DTI-derived indexes and total scores of MoCA as well as MMSE after controlling for age, sex and education.

\section{Discussion}

In our research, WMH patients were divided into two groups: one group with WMH score of 1-

2 points, and the other with $\mathrm{WMH}$ score of 3-6 points. We observed increased $\mathrm{MD}, \mathrm{AD}$ and $\mathrm{RD}$ in the bilateral anterior limb of internal capsule, bilateral posterior limb of internal capsule, bilateral retrolenticular part of internal capsule, bilateral posterior corona radiata, bilateral external capsule, bilateral superior longitudinal fasciculus and bilateral superior fronto-occipital fasciculus. According to previous research, increased $\mathrm{MD}, \mathrm{AD}$, and $\mathrm{RD}$ suggest injury associated with axonal injury and demyelination $[23,24]$. This injury was considered as the underlying mechanism of 
WMH caused by microvascular pathology [25].

Zeng noted that the Fazekas score of 3 was an important watershed in WMH, from which the participants began to show significant injury in white matter microstructures [26]. While there was no significant difference in microstructure integrity between the mild WMH group and the nonWMH group [26]. If we consider patients with WMH 1-2 points as controls, our results indicate that changes of WM microstructure integrity are symmetrically present in the projection fibers as well as some long association fibers in CSVD patients with WMH. Similar to our results, in the research of patients with subcortical ischemic vascular disease (SIVD) which was known as the most common type of CSVD, Liu found microstructural changes were extensive, mainly in the corpus callosum, bilateral inferior fronto-occipital fasciculus, superior longitudinal fasciculus, inferior longitudinal fasciculus, as well as the bilateral anterior thalamic radiation and corticospinal tract which are part of the inner capsule fibers [2]. Others pointed out that in patients with CSVD, the microstructural integrity of the projected fibers such as the inner capsule, the radiating crown, along with the post thalamic radiation, and the associated fibers like the bilateral superior longitudinal tract as well as the left inferior fronto-occipital fasciculus were impaired [27]. From another point of view, with the increase of WMH scores, the impairment of microstructures of the projection fibers as well as some long association fibers was more obvious. We hypothesized that these WM fibers were very sensitive to hemodynamic changes. The more severe the damage of WM microstructure was, the more obvious the WMH presented on conventional MRI.

In addition to the results above, we also found the decreased FA in the bilateral tapetum along with left posterior thalamic radiation, where increased $\mathrm{MD}, \mathrm{RD}$ and $\mathrm{AD}$ were not present. FA represents the normalized ratio of diffusion direction, reflecting the degree of arrangement of 
198

199

cellular structures in the fiber bundles and their structural integrity. The decreased FA value and increased MD value both reflect the gradual decrease of WM intensity. However, Liu concluded that the MD was more sensitive for the progression of cerebral small vessel disease compared with FA. Previous studies have also shown that $\mathrm{MD}$ and $\mathrm{RD}$ might serve as early markers of demyelination in WM regions $[28,29]$. As the development of WMH is partly caused by focal ischemia, which may lead to decreased tissue density and increased water diffusivity while maintaining the underlying directional structure, and these lead to an increase in MD with FA unchanged [30, 31]. Therefore, we focused on the areas where $\mathrm{MD}, \mathrm{AD}$, and $\mathrm{RD}$ values increased.

On the aspect of anatomy, the superior longitudinal fasciculus is the long bundle that connects the frontal, parietal, occipital, and temporal lobes. The frontal-occipital fasciculus connects the frontal lobes, occipital lobes, and temporal lobes. Projective fibers connect the cortex to other areas of the central nervous system by ascending fibers reaching the cortex and descending fibers leaving the cortex.

On the functional level, as fiber tracts that connect the cortex to the cortex, associative fiber tracts are the basis of cognitive integrity. Among them, superior longitudinal fasciculus connects sensory and motor language regions in the dominant hemisphere. Superior longitudinal fasciculus and inferior longitudinal fasciculus are the main associative fibers that connect the frontal parietal occipital cortex which is involved in executive function and processing speed [32]. Previous studies have shown that the MD index of bilateral inferior longitudinal fasciculus and right superior longitudinal fasciculus in the pre-SIVD patients was significantly positively correlated with the cognitive assessment. Besides, projective fibers play an important role in the basal-prefrontal circuitry of the hypothalamus. The anterior limb of internal capsule and anterior coronal radiations 
are the main projecting fibers between the frontal cortex and thalamus [33]. The deterioration of WM in these regions supports the involvement of subcortical circuits in the development of CSVD related cognitive impairment [34].

All of these suggest that with the increase of the severity of $\mathrm{WMH}$, the impairment of microstructure tends to occur on the WM fiber tracts which are closely related to cognition. Similarly, researchers noticed that in the early stage of CSVD, WM microstructural injury mainly occurred in the cognition-related WM fibers [35-36]. Therefore, we hypothesized that in CSVD patients, the WM fiber tracts, which are closely related to cognition, are more susceptible to be injuried. This may be the reason why CSVD patients often suffer from cognitive impairment.

However, in this study, we found no correlation between the DTI derived index and cognition, which was inconsistent with previous studies $[14,16,17,39]$. This may be related to the fact that our enrolled subjects are mostly preclinical patients. These subjects tended to show only microstructure impairment but not obvious clinical symptoms such as cognitive decline. Previous study indicated that in these non-dementia CSVD patients, only a few areas showed significant node efficiency changes which contribute to cognition decline, despite extensive WM integrity impairment [27]. For one reason, WMH represents loss of the myelin sheath and axon and does not cause complete destruction of the fibers especially in the early stage of CSVD. For the other, reactive structural plasticity such as gliosis is a common histopathological change in CSVD, which may lead to the strengthening of interhemispheric connections [40]. Hence, we conclude that changes in WM microstructure in CSVD patients predate cognitive decline. If we can detect the microstructural changes in WM before the onset of cognitive decline and give some interventions, we may be able to delay cognitive decline to some extent. 

study which limits our observation on longitudinal effects of cerebral small vessel disease. Secondly, patients with WMH were graded by visual observation, which is somewhat subjective and cannot accurately reflect the severity of white matter lesions (WMLs). Finally, no health control group was set up in our research. Therefore, we plan to include healthy subjects in further experimental study. Besides, we will assess the WMH load by measuring the WMH volume as well as its location.

\section{Conclusion}

In CSVD patients, the WM fiber tracts that are closely related to cognitive function tend to be more vulnerable to be injured, and the injury of these WM fiber tracts is more obvious with the aggravation of WMH degree. In addition, changes in WM microstructure often predate changes of cognition. Therefore, early detection of microstructural changes and timely intervention can delay cognitive decline to some extent.

Ethics approval and consent to participate

\section{Consent for publication} Not applicable.

\section{Availability of data and materials}




\section{Funding}

\section{Authors' contributions} submitted to the journal. All authors read and approved the final manuscript.

\section{Acknowledgement}

275 We would like to gratefully thank Pro. Chi-Shing ZEE, Keck hospital of USC and Xiao Liu for their kindly revised the manuscript finally.

\section{References:}

278 1. $\mathrm{Xu} \mathrm{X}$ et al. Progression of White Matter Hyperintensities Contributes to Lacunar Infarction. Aging Dis

$279 \quad 2018 ; 9: 444-52$. Cognitive Impairment: a TBSS Study. J Mol Neurosci 2019;67:595-603. Rev Neurol 2015;11:157-65. 
288 6. Zeestraten EA et al. Change in multimodal MRI markers predicts dementia risk in cerebral small vessel disease. Neurology 2017;89:1869-76. sensitivity to change over time. Stroke 2008;39:1999-2005. in patients with subcortical ischemic vascular disease. Dement Geriatr Cogn Disord 2010;30:317-26. contribute toward working memory performance in older adults. Neuroreport 2016;27:689-93. 
308

16. De Lange AG et al. The effects of memory training on behavioral and microstructural plasticity in young and older adults. Hum Brain Mapp 2017;38:5666-80.

17. Mascalchi $\mathrm{M}$ et al. DTI-derived indexes of brain WM correlate with cognitive performance in vascular $\mathrm{MCl}$ and small-vessel disease. A TBSS study. Brain Imaging Behav 2019;13:594-602.

18. Fazekas $\mathrm{F}$ et al. MR signal abnormalities at $1.5 \mathrm{~T}$ in Alzheimer's dementia and normal aging. AJR Am J Roentgenol 1987;149:351-6.

19. Smith SM. Fast robust automated brain extraction. Hum Brain Mapp 2002;17:143-55.

20. Sun Y, Zhou M, Wang D, et al. Application of Echocardiography Information Technology in Monitoring Cardiac Function and Peripheral Neurotoxicity of HPV Patients Infected with Breast Cancer after Chemotherapy. Neuroscience Letters 2020;135210.

21. Heney N M, Ahmed S, Flanagan M J, et al. Superficial bladder cancer: progression and recurrence. The Journal of urology 1983; 130(6): 1083-1086.

22. Gelman A, Loken E. The statistical crisis in science: data-dependent analysis--a" garden of forking paths"-explains why many statistically significant comparisons don't hold up. American scientist 2014; 102(6): 460-466.

23. Huang $\mathrm{J}$ et al. White matter microstructural alterations in clinically isolated syndrome and multiple sclerosis. J Clin Neurosci 2018;53:27-33.

24. Sun Y et al. Cerebral Blood Flow Alterations as Assessed by 3D ASL in Cognitive Impairment in Patients with Subcortical Vascular Cognitive Impairment: A Marker for Disease Severity. Front Aging Neurosci 2016;8:211.

25. Joutel A, Chabriat H. Pathogenesis of white matter changes in cerebral small vessel diseases: beyond vesselintrinsic mechanisms. Clin Sci (Lond) 2017;131:635-51.

26. Zeng $\mathrm{W}$ et al. Severity of white matter hyperintensities: Lesion patterns, cognition, and microstructural changes. Journal of Cerebral Blood Flow \& Metabolism 2019:271678X-1989360X. 
332 28. Hulst HE et al. Cognitive impairment in MS: impact of white matter integrity, gray matter volume, and lesions. mild cognitive impairment, and Alzheimer's patients. Neurol India 2011;59:168-73. aging. Neuroimage Clin 2013;3:180-95. Neuroimage 2008;40:570-82. 
353 39. Otsuka Y et al. Diffuse tract damage in the hemispheric deep white matter may correlate with global cognitive

354 impairment and callosal atrophy in patients with extensive leukoaraiosis. AJNR Am J Neuroradiol 2012;33:726-32.

355 40. Pantoni L. Cerebral small vessel disease: from pathogenesis and clinical characteristics to therapeutic challenges. Lancet Neurol 2010;9:689-701.

357

358

359

360 


\section{Figures}

\section{Differences in axial diffusion (AD) values of white matter fiber bundles between groups}

$\mathbf{L}$
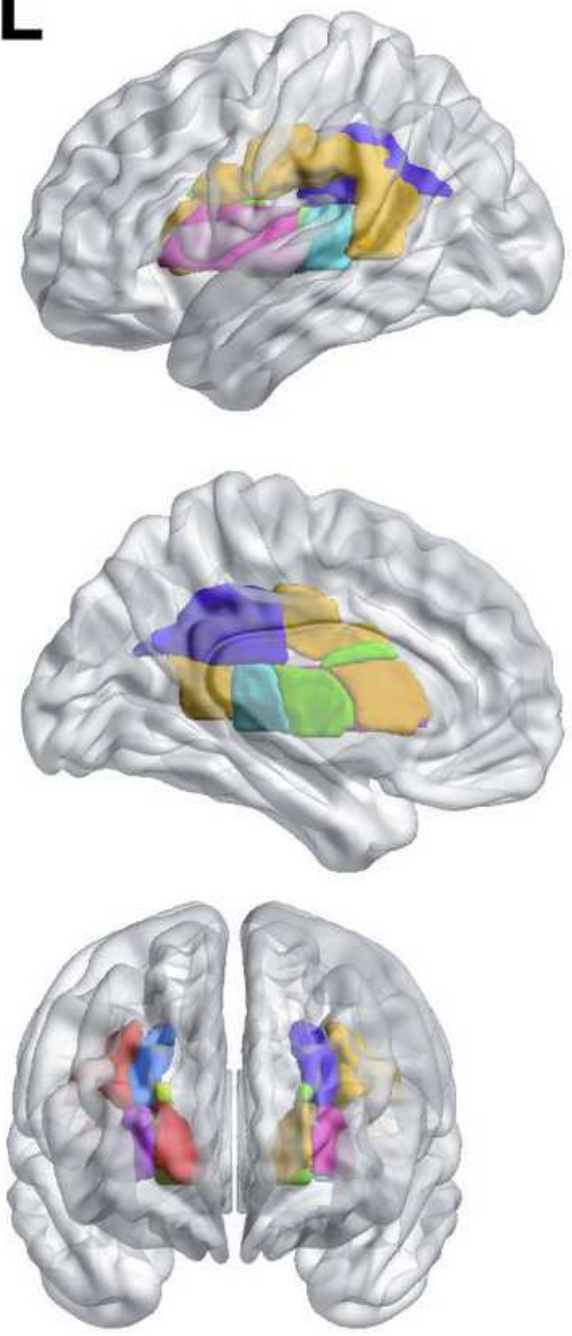
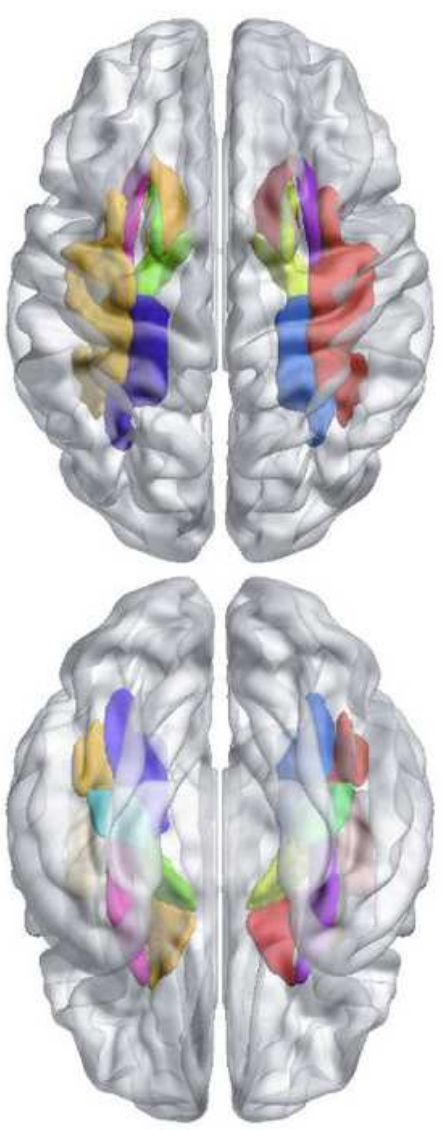

$\mathbf{R}$
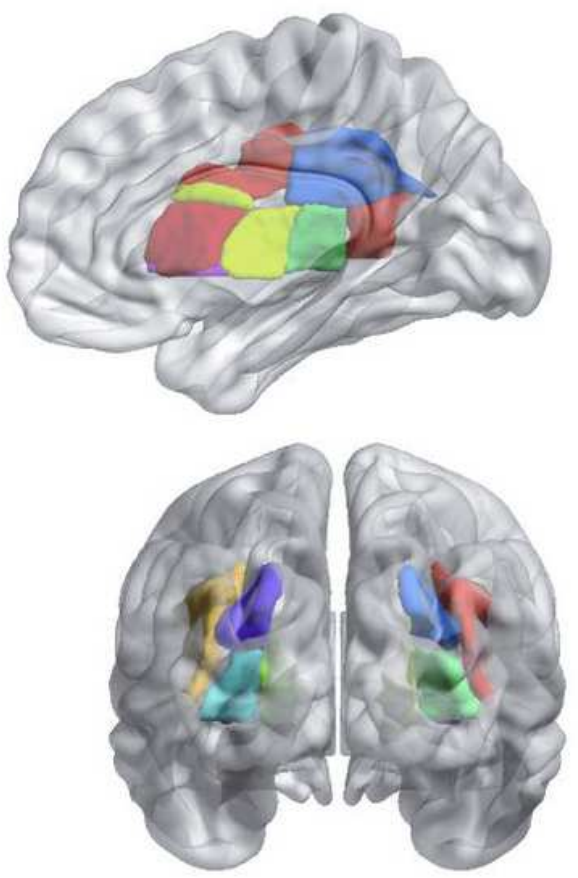

Figure 1

Different axial diffusion (AD) values of white matter fiber bundles between two groups are marked in colors. Patients in Group B had higher AD values of some white matter fiber bundles than those in group A. 
Differences in fractional anisotropy (FA) values of white matter fiber bundles between groups

$\mathbf{L}$
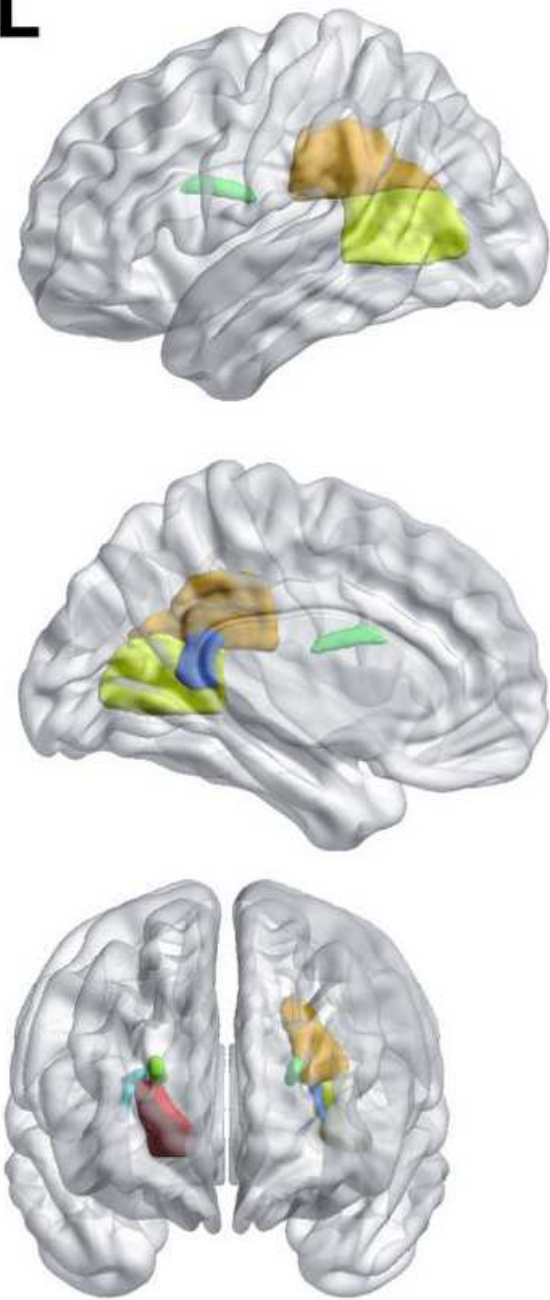
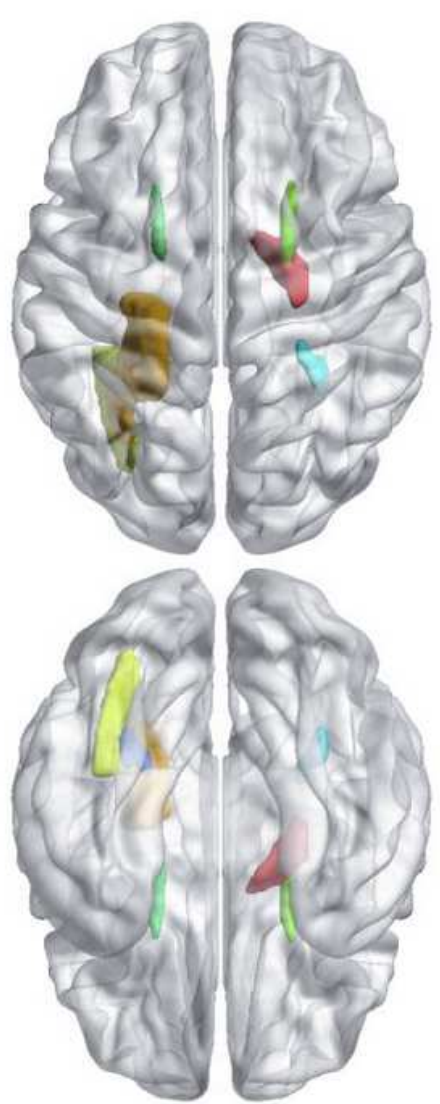

$\mathbf{R}$
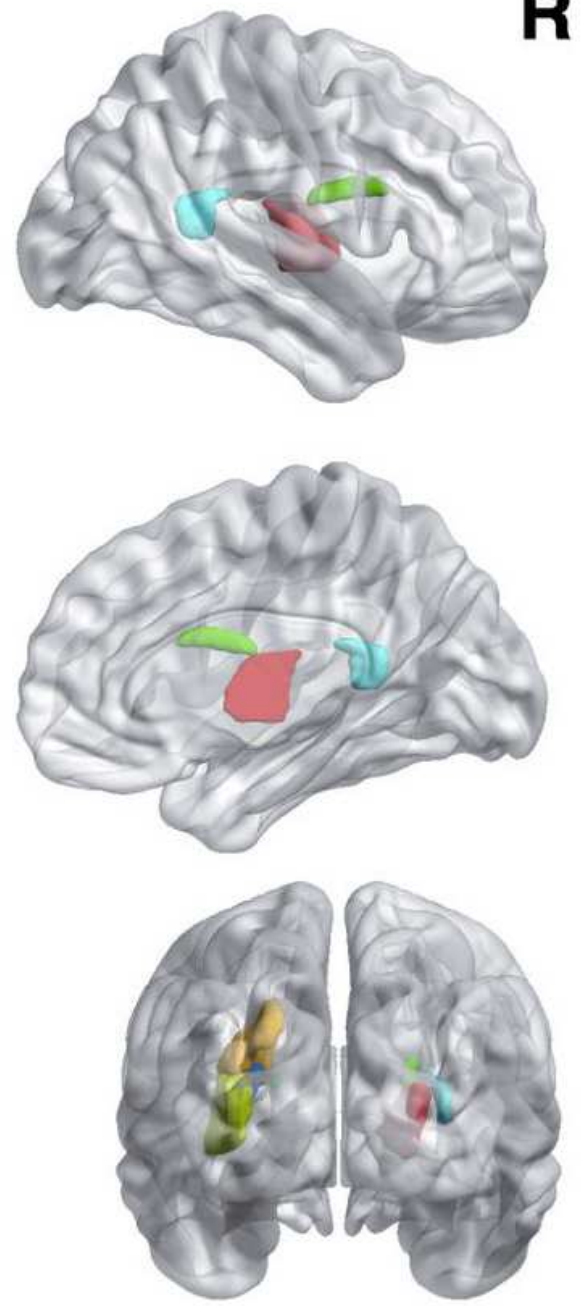

Figure 2

Different mean diffusion (MD) and radial diffusion (RD) values of white matter fiber bundles between two groups are marked in colors. Patients in Group B had higher MD and RD values of some white matter fiber bundles 158 than those in group $A$. 


\section{Differences in mean diffusion (MD) and radial diffusion (RD) values of white matter fiber}

\section{bundles between groups}
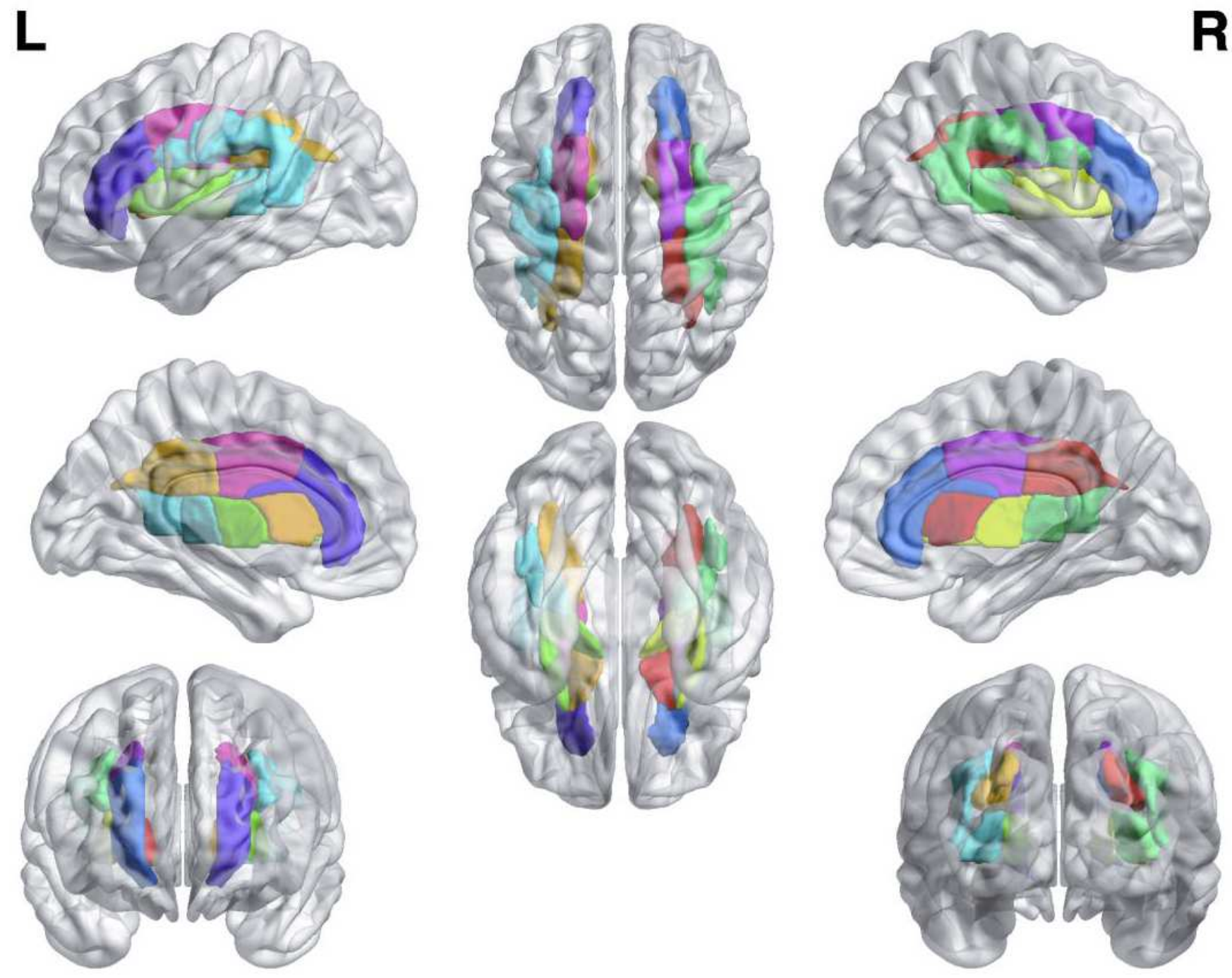

Figure 3

Different fractional anisotropy (FA) values of white matter fiber bundles between two groups are marked in colors. Patients in Group B had lower FA values of some white matter fiber bundles than those in group A.

\section{Supplementary Files}

This is a list of supplementary files associated with this preprint. Click to download.

- Table.pdf 